\title{
Effect of Seed Heat-Treatment on the Oxidative Stability of Canola Oil Body Emulsions
}

\author{
Zhiping Shen, Chakra Wijesundera, Jian-Hui Ye \\ Food and Nutritional Sciences, Commonwealth Scientific and Industrial Research Organization, Victoria, Australia. \\ Email: chakra.wijesundera@csiro.au
}

Received May $1^{\text {st }}, 2012$; revised June $1^{\text {st }}, 2012$; accepted June $8^{\text {th }}, 2012$

\begin{abstract}
Enhancement of oxidative stability of canola oil extracted from seed subjected to prior heat-treatment has been attributed to heat-induced generation of antioxidants from phenolic precursors occurring in canola seed. Dispersion of aqueous extracts of intact seed oil bodies (OBs) in water is a novel and interesting way of producing natural and oxidatively stable food emulsions with minimal use of synthetic antioxidants and emulsifiers. As there is growing interest in natural food emulsions containing unsaturated oils, we investigated whether the oxidative stability of canola $\mathrm{OB}$ emulsions could be further improved by subjecting canola seed to heat-treatment prior to oil body extraction. Oil-in-water (5\%, $\mathrm{w} / \mathrm{w}$ ) emulsions of OBs extracted from canola seed before and after heat-treatment were considerably more resistant to oxidation than emulsions prepared from refined canola oil and Tween ${ }^{\circledR} 40$ emulsifier. However, only small amounts $(0.9 \%-4.5 \%$ by weight) of the phenolic compounds present in canola seed were transferred to the OBs after aqueous extraction, and consequently there was no discernible effect on oxidative stability as a result of prior heat-treatment of the seed. Thus, in contrast to oil, there is no oxidative stability benefit to be gained by subjecting canola seed to heattreatment prior to extraction of OBs.
\end{abstract}

Keywords: Canola Seed Heat-Treatment; Food Emulsions; Oil Bodies; Oxidative Stability; Phenolic Antioxidants

\section{Introduction}

Triacylglycerols (TAGs) are stored within oilseeds in the form of discrete spherical deposits referred to as oil bodies $(\mathrm{OBs})$. They provide energy and carbon skeletons required for germination and subsequent seedling growth $[1,2]$. OBs from diverse species of oilseeds are very similar in structure [3] and contain a TAG core surrounded by a monolayer of phospholipids (PL) embedded with proteins [4]. Although variable between species, the size of OBs falls within a narrow range of approximately $0.5-2.0 \mu \mathrm{m}$ in diameter despite wide variation in the total oil content in the tissue. This size may represent a balance between the conflicting requirements for efficient packaging and mobilization during germination, the latter being facilitated by a high surface to volume ratio [2].

A special feature of OBs is their remarkable resistance to aggregation both inside cells and in isolated preparations [1,5]. In both situations, OBs occur as individual entities, and do not aggregate or coalesce when they are pressed against one another even after prolonged storage [5]. This high stability within the oilseed is required to withstand extremes of desiccation, rehydration, heating and cooling for months or even years before the storage oil can be mobilized following seed germination [6].

The exceptional physical stability of OBs has been attributed to unique amphipathic proteins called oleosins that occur within the oil body surface [4,5]. They are thought to stabilize OBs by causing steric hindrance and electronegative repulsion $[4,6]$. It has been suggested that the entire surface of the OB is covered by oleosin such that the compressed OBs never coalesce or aggregate in the cells of a mature seed [5]. It has also been shown that in corn, soybean and rape, TAGs and oleosins accumulate concomitantly [3]. The content of oleosins in mature seeds ranges from $1 \%-4 \%$ of the total OBs; the greatest proportion occurring in species such as rape/canola where the OBs are the smallest $[3,7]$.

The storage of TAGs within OBs not only prevents the TAG droplets from aggregation and coalescence but also appears to protect them from lipid oxidation. OBs protect lipid reserves against oxidation and hydrolysis until seed germination and seedling establishment [8]. Although the concentrations of unsaturated fatty acids in whole soybean seeds were unchanged during accelerated aging [9], a marked decline was observed after extended natural aging [10] possibly due to destabilisation of OBs by lipolysis of phospholipids at the OB surface [11]. Recent 
studies have shown that aqueous dispersions of intact OBs extracted from sunflower [12] and echium seeds [13] are more resistant to oxidative deterioration compared to equivalent emulsions of extracted canola oil stabilized with added emulsifier. Oils of sunflower and echium contain highly unsaturated fatty acids. Such acids are very susceptible to oxidative deterioration resulting in a rapid decrease in palatability, nutritional quality and shelf-life of foods into which they are incorporated. Intact OBs offer a novel and effective route to the preparation of oxidatively stable food emulsions from such unstable oils without necessarily using synthetic antioxidants for stabilization.

Current international dietary guidelines advocate replacement of saturated fats in food with unsaturated fats [14] which heightens the need to develop more efficient and improved methods for stabilisation of unsaturated oils incorporated in to emulsion-based foods. On account of its very low content of saturated fatty acids and high content of unsaturated fatty acids, canola oil is one of the healthiest oils available for food use. However, the presence of polyunsaturated fatty acids (PUFA) makes food emulsions containing canola oil susceptible to oxidative deterioration resulting in loss of palatability of the product, and consequently the product shelf-life. On the other hand, canola seed has a greater abundance of phenolic compounds compared with most other oilseeds [15] that can potentially be used as natural antioxidants. Previous studies have shown that the antioxidant potential of canola phenolics can be activated by subjecting the seed to heat treatment prior to oil extraction [16]. The enhanced oxidative stability of canola oil extracted from heattreated seed has been attributed to 4-vinyl-2,6-dimethoxyphenol (vinyl syringol) produced by thermal decarboxylation of sinapic acid derived from sinapine occurring in canola seed [17,18].

It is not known whether the oxidative stability of canola OBs could be further improved, in the same way as the oxidative stability of bulk canola oil is improved, by subjecting the seed to heat treatment prior to extraction of OBs. Here we report on the oxidative stability of aqueous dispersions of intact OBs isolated from heated and unheated canola seeds relative to that of oil in water emulsions prepared from refined canola oil and Tween ${ }^{\circledR}$ 40 emulsifier. We also describe the transfer routes of phenolic compounds during the aqueous extraction process typically used to extract OBs from canola seed.

\section{Experimental}

\subsection{Materials}

Canola seed (Brassica napus) was provided by Cargill Australia from a batch of seed prepared for commercial oil extraction. Cargill Australia also supplied refined, bleached and deodorized oil extracted from the same batch of canola seed. The oil contained no added antioxidants. Sodium azide $\left(\mathrm{NaN}_{3}, 99.9 \%\right.$ purity), Tween ${ }^{\circledR}$ 40 (polyoxyethylene sorbitan monopalmitate) and all solvents (HPLC grade) were purchased from Sigma-Aldrich (Sydney, Australia).

\subsection{Heat Treatment of Canola Seed}

Canola seeds were evenly spread on a stainless steel tray and placed inside in an oven (Rational, Combi-Dampfer, Swann Drive, Derrimut, VIC, Australia) preheated to $160^{\circ} \mathrm{C}$. The tray was removed from the oven for $7.5 \mathrm{~min}$ after the temperature of the seed reached and stabilized at the set temperature.

\subsection{Extraction of Canola Oil Bodies}

Canola seeds (fresh or heat-treated, $125 \mathrm{~g}$, on dry weight basis) were soaked overnight in deionised water and homogenized with further deionised water $(500 \mathrm{~mL})$ using a kitchen blender (Sunbeam Multiblender Platinum, PB7610). The blending was carried out successively for $20 \mathrm{~s}, 40 \mathrm{~s}$, and $2 \mathrm{~min}$ at the first, second, and the highest speed settings, respectively. After the addition of a further amount of deionised water, the blending was continued at the highest speed setting for another $30 \mathrm{~s}$, and the slurry was filtered through three layers of cheese cloth. The filter cake was washed with more deionized water and pressed. The total amount of water used for washing was $250 \mathrm{~mL}$.

The filtrate was placed in $2 \times 400 \mathrm{~mL}$ centrifuge tubes, centrifuged at $9500 \mathrm{rpm}$ for $50 \mathrm{~min}$ (JA-10 rotor, Beckman, J2-MC, Beckman Coulter, Inc., Brea, CA, USA), and the creamy top layer was collected. It was washed twice by suspending in $0.2 \mathrm{M}$ aqueous sodium chloride followed by centrifugation to recover the washed cream pads. The entire procedure of collection of the top cream pad, dispersion, and centrifugation was repeated one more time except that a solution of $0.02 \%$ aqueous $\mathrm{NaN}_{3}$ was added prior to the final centrifugation to prevent microbial growth during acceleration oxidation studies. The extraction procedure was repeated three times, the cream pads pooled, and kept at $4^{\circ} \mathrm{C}$ until required for emulsion preparations. The average dry weights (per extraction) of the top cream pads from heated and unheated seed were $20.3 \pm 0.2 \mathrm{~g}$ and $28.1 \pm 0.4 \mathrm{~g}$, respectively.

\subsection{Gross Composition of Oil Bodies}

The moisture content of the OBs was determined by loss of weight after drying to constant weight at $105^{\circ} \mathrm{C}$. The fat content was determined using a modification of the method described by Christie [19] in which an iso-propanol (IPA) to hexane ratio of $1: 1(\mathrm{v} / \mathrm{v})$ was used instead 
of 3:2. The protein content was calculated from the nitrogen content measured using an automated nitrogen analyzer LECO model FP-2000 (LECO Corporation, Joseph, Michigan, USA). The ash content was determined after heating a sample of OBs inside a muffle furnace at $600^{\circ} \mathrm{C}$ for $5 \mathrm{~h}$. The extraneous matter was calculated from the difference in weights.

\subsection{Preparation of Oil in Water Emulsions}

Measured amounts of OBs extracted from heated and unheated canola seeds were dispersed in deionised water to obtain $5 \%(\mathrm{w} / \mathrm{w})$ oil-in-water emulsions. The $\mathrm{pH}$ of the resulting dispersion was 6.1. Although some flocculation was observed probably due to aggregation of OBs at this $\mathrm{pH}$, no coalescence occurred during storage. An oil-in-water emulsion with the same oil content was prepared with refined canola oil and Tween ${ }^{\circledR} 40$ emulsifier $(0.5 \% \mathrm{w} / \mathrm{w})$ by mixing measured amounts of oil, water and emulsifier using a Ultra-Turrax mixer (Model T25 Janke \& Kunkel, Stafen, Germany) which was operated at $9500 \mathrm{rpm}$ for $5 \mathrm{~min}$ followed by $13,500 \mathrm{rpm}$ for a further $5 \mathrm{~min}$. No antioxidants were added to the emulsions.

\subsection{Electrophoretic Analysis}

Electrophoresis was performed with a NuPAGE containing 4\% - 12\% BT 1.0 (NuPAGE ${ }^{\circledR}$ Invitrogen) according to supplier recommendations. Aqueous dispersions of OBs $\left(1 \mathrm{mg} \cdot \mathrm{mL}^{-1}, 25 \mu \mathrm{L}\right)$ were placed in Eppendorf tubes to which NuPAGE ${ }^{\circledR}$ LDS sample buffer $(10 \mu \mathrm{L})$ and NuPAGE ${ }^{\circledR}$ reducing agent $(5 \mu \mathrm{L})$ were added. The tubes were centrifuged (Eppendorf Centrifuge 5415C) for $3 \mathrm{~s}$ at $14,000 \mathrm{rpm}$ before being heated at $70^{\circ} \mathrm{C}$ for $10 \mathrm{~min}$. Markers (Mark 12 ${ }^{\mathrm{TM}}$ unstained standards) were prepared as per the samples. Finally, both markers and samples (10 $\mu \mathrm{L}$ each) were loaded into the gel, which was already placed in an electrophoresis cell (Novex Mini-Cell, Invitrogen) filled with adequate amounts of NuPAGE ${ }^{\circledR}$ running buffer and antioxidant according to supplier recommendations. The cell was connected to a generator (BIORAD Power Pac 300) set to $200 \mathrm{~V}$ and $400 \mathrm{~mA}$ for $35 \mathrm{~min}$. Following electrophoresis, the gel was washed 3 times in deionised water and placed in a plastic container with SimplyBlue ${ }^{\text {TM }}$ SafeStain (Invitrogen) for $2 \mathrm{~h}$ under slow agitation (42 rpm, RATEK Platform Mixer model OM6). Afterwards, the gel was washed twice with deionised water and placed in deionised water for a further $2 \mathrm{~h}$ in order to dilute the stain remaining in the gel and not bound to proteins. Once the gel was free of non protein-bound stain an image was taken using a G:BOX and GeneSnap 7.07 software (SYNGENE).

\subsection{Oxidative Stability of Oil Body Emulsions}

Samples of emulsions $(2 \mathrm{~mL})$ were placed in glass head- space vials $(10 \mathrm{~mL})$ and sealed by aluminium caps fitted with Teflon-coated silica septa. The vials were stored inside a dark oven maintained at $60^{\circ} \mathrm{C}$ to accelerate oxidation. Vials were withdrawn at periodic intervals and two vials were immediately subjected to headspace analysis. Another two vials were frozen at $-20^{\circ} \mathrm{C}$ until required for peroxide value (PV) and fatty acid profile determinations.

\subsection{Headspace Analysis}

Volatile oxidation products within emulsions (triplicate sample vials) were measured using headspace solid phase micro extraction (SPME) combined with gas chromatography-mass spectrometry (GC-MS). The SPME fiber (DVB/Car/PDMS, 50/30 $\mu \mathrm{m}$; Supelco, Sydney, Australia) was inserted into the sample headspace and the vial was incubated at $60^{\circ} \mathrm{C}$ for $15 \mathrm{~min}$. The fiber was then withdrawn and transferred to the GC injector (operated in the splitless mode) and held there for $7 \mathrm{~min}$ to desorb the extracted volatile compounds into the GC column. The entire series of events was performed using a CombiPAL Auto Injector (CTC Analytics, Zwingen, Switzerland). GC-MS was performed using an Agilent Model 6890 GC and Model 5973 mass spectral detector (Palo Alto, CA) fitted with a VOC fused silica capillary column $(60 \mathrm{~mm}$, $0.32 \mathrm{~mm}$ i.d., $0.18 \mu \mathrm{m}$ film thickness, Agilent, Melbourne, VIC, Australia). The GC oven was programmed from $40^{\circ} \mathrm{C}$ to $220^{\circ} \mathrm{C}$ at the rate of $22^{\circ} \mathrm{C} \mathrm{min}^{-1}$ and held at that temperature for a further $14 \mathrm{~min}$. Helium was used as the carrier gas at a constant flow rate of $2 \mathrm{~mL} \cdot \mathrm{min}^{-1}$. The injector was initially operated in the splitless mode and then switched to the split mode (1:20) 2 min after sample injection. The temperature of the injector and the MS detector were both held at $230^{\circ} \mathrm{C}$. The MS was operated in the scan mode (29 - $250 \mathrm{amu})$. Data analyses were performed using Chemstation software and compounds were identified by comparison with standards as well as reference to a library of spectra (Wiley 275). Concentrations of the volatile compounds within emulsions were calculated using calibration curves established by spiking fresh emulsions with different levels of standards representing the compounds being measured.

\subsection{Recovery of Oil from Emulsions}

Water $(1 \mathrm{~mL})$ and IPA $(4 \mathrm{~mL})$ were added to the headspace (HS) vial containing emulsion $(2 \mathrm{~mL})$, tightly sealed, and vigorously vortexed for $30 \mathrm{~s}$ before transferring the mixture into a clear glass culture tube $(15 \mathrm{~mL})$. The empty vial was rinsed with hexane $(4 \mathrm{~mL})$ and the wash was added to the culture tube, which was then centrifuged at $3000 \mathrm{rpm}$ for $15 \mathrm{~min}$ (J6-HC, Beckman) at ambient temperature. The top solvent layer (a mixture of hexane and small amount of IPA) was withdrawn into 
another $15 \mathrm{~mL}$ clear glass culture tube. A further amount of hexane $(4 \mathrm{~mL})$ was added to the first culture tube and vigorously vortexed until the solid plug between the two phases was completely disintegrated into fine homogeneous pieces. The top hexane layer was recovered and combined with the first extract. The extraction was repeated one more time and the pooled hexane extracts were concentrated under nitrogen. After complete removal of solvent, hexane ( $8 \mathrm{~mL}$, accurately measured) was added to the recovered oil and the tube was vortexed to obtain a solution of oil in hexane. The modified extraction method was in good agreement with oil contents determined by the Bligh \& Dyer method [20]; oil recovered from canola OBs by this method $(36.88 \% \pm 1.08 \%)$ was practically the same as that obtained by the Bligh \& Dyer method $(36.77 \% \pm 0.97 \%)$.

\subsection{Peroxide Value (PV)}

PV of oil extracted from the emulsions (duplicate samples) was determined using a SāfTest ${ }^{\circledR}$ Analyzer (SāfTest ${ }^{\circledR}$, Inc, USA). In this colorimetric test, the oil sample is treated with three different reagents supplied by the manufacturer of the instrument, and UV absorbance (570 $\mathrm{nm}$ filter) recorded with an UV analyzer. The PV of the test samples was then calculated by reference to a calibration curve obtained with PeroxySafe ${ }^{\mathrm{TM}}$ calibrating reagent supplied by the manufacturer.

\subsection{Fatty Acid Analysis}

The extracted oils (duplicate samples) were converted to fatty acid methyl esters by alkali-catalysed transesterificaton and analyzed by capillary GC analysis as previously described [16].

\subsection{Determination of Phenol Content}

Ground canola seed $(4 \mathrm{~g})$ was extracted three times with $70 \%(\mathrm{v} / \mathrm{v})$ ethanol $(40 \mathrm{~mL})$, centrifuged at $9500 \mathrm{rpm}$ for $15 \mathrm{~min}$ and the supernatant was collected. The supernatant was diluted 10 -fold with pure water before measuring the phenol content using the Folin-Ciocalteu's assay as follows: in brief, the diluted extract $(100 \mu \mathrm{L})$ and the serial standard solutions of gallic acid were loaded on to a 96-well microplate. Folin-Ciocalteu's phenol reagent $(80 \mu \mathrm{L})$ was added and allowed to stand for $3 \mathrm{~min}$. Next, sodium carbonate solution $(7.5 \% \mathrm{w} / \mathrm{v}, 120 \mu \mathrm{L})$ was added and the contents were mixed well and allowed to stand in the dark for a further $40 \mathrm{~min}$ when the absorbance of the mixture at $765 \mathrm{~nm}$ was recorded using the microplate reader.

As it was impractical to extract phenols from OBs using $70 \%(\mathrm{v} / \mathrm{v})$ ethanol due to emulsion formation, OBs were first separated in to their components (TAG, PL and membrane material) according to the method of Tzen and Huang [5], and the phenol concentration of each fraction was measured separately. The phenol contents of the OBs were calculated indirectly by summing the component concentrations. The OBs were extracted first with diethyl ether to recover the TAGs followed by dichloromethane/methanol $(2: 1, \mathrm{v} / \mathrm{v})$ to recover the PLs. The TAGs were diluted 1:1 (v/v) with hexane and extracted three times with $50 \%(\mathrm{v} / \mathrm{v})$ ethanol to extract phenols. The extract was diluted 4-fold with water, and the phenol content measured as described above for seed. Phenols were extracted from the filter cake and interfacial material using a method similar to that described for seed. All extractions were performed in triplicate.

\subsection{Statistical Analysis}

Statistical analyses were performed using Excel software (Microsoft Office, Windows 7).

\section{Results and Discussion}

Phenolic compounds are more abundant in canola than in any other oil seed [15]. The canola phenolics predominantly consist of derivatives of sinapic acid with sinapine as the main constituent $[15,21]$. The superior oxidative stability of oil from heat-treated canola seed has been attributed to vinyl syringol, which is thought to be formed by thermal degradation of sinapic acid $[16,18,22]$. It has been reported that the highest amount of vinly syringol was produced when the seed was heated to $160^{\circ} \mathrm{C}$ for 7.5 min giving a vinyl syringol content which is 120 -fold higher than that found in the unheated seed [23]; further heating, and heating at temperatures higher than $160^{\circ} \mathrm{C}$ was reported to result in considerable loss of vinyl syringol. This optimal temperature and time combination of $160^{\circ} \mathrm{C}$ for $7.5 \mathrm{~min}$ was used in the present study to heattreat the canola seed.

The moisture contents of OBs extracted from the heattreated (HOB) and unheated (UHOB) canola seeds were $36.7 \%$ and $42.6 \%(\mathrm{w} / \mathrm{w})$, respectively (Table 1$)$. On a moisture-free basis, the oil content of HOB $(85.0 \%)$ was higher than of UHOB $(81.7 \%)$ while the protein contents were comparable $(9.8 \%$ and $9.4 \%$, respectively), suggesting more efficient removal of the non-fat and nonprotein material during extraction of OBs from the heated seed. The oil recovered from both HOB and

Table 1. Gross composition of oil body preparations from heated $\left(160^{\circ} \mathrm{C}\right.$ for $\left.7.5 \mathrm{~min}\right)$ and unheated canola seed.

\begin{tabular}{cccccc}
\hline & \multicolumn{4}{c}{ Composition of canola oil body preparation (weight \%) } \\
\cline { 2 - 6 } & Oil & Moisture & Protein & Ash & Others \\
\hline Unheated & $46.9 \pm 0.2$ & $42.6 \pm 0.0$ & $5.4 \pm 0.0$ & $0.6 \pm 0.0$ & 4.5 \\
Heated & $53.8 \pm 0.1$ & $36.7 \pm 0.1$ & $6.2 \pm 0.1$ & $0.2 \pm 0.0$ & 3.1 \\
\hline
\end{tabular}


UHOB contained higher levels of the PUFA linoleic $(20.8 \%$ - 20.9\%) and linolenic $(11.4 \%$ - 11.8\%) compared with the refined oil produced commercially from the same batch of seed $(19.2 \%-19.3 \%$ and $8.1 \%$, respectively, Table 2). The fatty acid profiles observed for the oil recovered from the freshly extracted OBs represent the true fatty acid composition of canola. The observed reduction in the PUFA content in the comercially refined oil probably results from the loss of a small amount of these acids due to oxidative deterioration during industrial extraction and refining of oil.

The proteins isolated from the canola OBs predominantly consisted of components of molecular weight in the range 18 - $20 \mathrm{kDa}$. In all probability, they represented oleosins as the molecular weight of canola oleosins has been reported to be within this range [24]. SDS-PAGE analysis showed the presence of other protein components covering a wide range of molecular weights, albeit at relatively small concentrations (Figure 1). Although the protein compositions of OBs from the heated and unheated seeds were qualitatively similar, a higher abundance of components with molecular weight greater than $40 \mathrm{kDa}$ was observed for the OBs extracted from the unheated seed.

The oxidative stabilities of aqueous dispersions of OBs extracted from heated and unheated canola seed were compared with an emulsion prepared from refined canola oil and Tween ${ }^{\circledR} 40$ emulsifier using three independent evaluation methods. Tween ${ }^{\circledR} 40$ was selected as the emulsifier for canola oil for two main reasons. Firstly, it is a food-grade emulsifier, and secondly, emulsions made using non-ionic emulsifiers such as Tween ${ }^{\circledR} 40$ are more stable to oxidation than those made using anionic or cationic emulsifiers [25]. Oxidation was performed at $60^{\circ} \mathrm{C}$ to accelerate oxidation; temperatures between $40^{\circ} \mathrm{C}$ and $60^{\circ} \mathrm{C}$ are deemed suitable for accelerating oxidation of vegetable oil-based systems without causing too excessive oxidation beyond the point at which rancid flavours are detected [26].

Figure 2 shows the development of hydroperoxides, as measured by PV, at different accelerated oxidation times. The PV of the canola oil/Tween ${ }^{\circledR} 40$ emulsion increased sharply over the 8 day accelerated oxidation without an apparent induction period. In contrast, the PV of the OB dispersions from heated and unheated seed remained practically static during this time showing that emulsions prepared from intact canola OBs are more resistant to oxidative deterioration compared with emulsions made from refined canola oil and Tween ${ }^{\circledR} 40$ emulsifier.

The superior oxidative stabilities of canola OB emulsions over canola oil/Tween ${ }^{\circledR} 40$ emulsion was further substantiated by the difference in the rate of formation of trans, trans-2,4-heptadienal during accelerated oxidation. This compound is an oxidation product of linolenic acid, and is a reliable marker of oxidation of omega-3 oils. Its concentration increased rapidly for the canola oil/Tween ${ }^{\circledR}$ 40 emulsion and very slowly for both of the OB dispersions (Figure 3). A similar trend was observed for hexanal, which is an oxidation product of linoleic acid (results not shown). Further evidence for the superior oxidative stability of emulsions made from intact canola OBs came from the depletion rates of linoleic and lino-

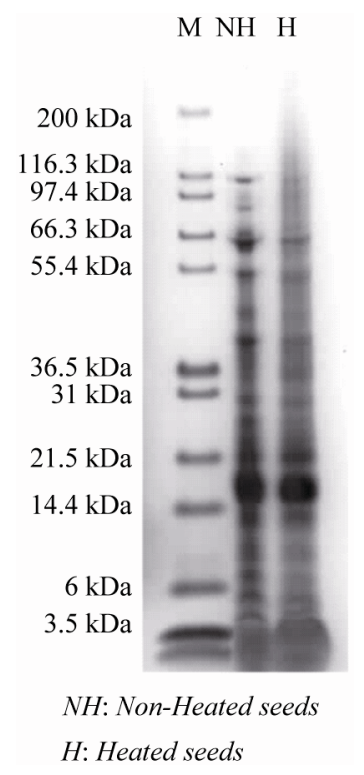

Figure 1. Sodium dodecyl sulphate-polyacrylamide gel electrophoresis (SDS-PAGE) of protein separated from canola oil bodies. M, molecular markers; NH, oil bodies from nonheated seed; $\mathrm{H}$, oil bodies from heat-treated seed.

Table 2. Fatty acid composition of commercial canola oil and oils recovered from canola oil/Tween 40 emulsion and aqueous dispersions of oil bodies extracted from canola seed before and after heat treatment $\left(160^{\circ} \mathrm{C}\right.$ for 7.5 min). Fatty acid concentration is expressed as a weight percentage of total fatty acids (average values for duplicate samples).

\begin{tabular}{|c|c|c|c|c|c|c|c|c|}
\hline Sample & $16: 0$ & 18:0 & $\begin{array}{c}18: 1 \\
n-9\end{array}$ & $\begin{array}{c}18: 1 \\
n-7\end{array}$ & $\begin{array}{c}18: 2 \\
n-6\end{array}$ & $\begin{array}{c}18: 3 \\
n-3\end{array}$ & 20:0 & $20: 1$ \\
\hline Refined canola oil & $4.4 \pm 0.0$ & $2.2 \pm 0.0$ & $60.4 \pm 0.0$ & $3.0 \pm 0.0$ & $19.3 \pm 0.6$ & $8.1 \pm 0.0$ & $0.6 \pm 0.0$ & $1.0 \pm 0.0$ \\
\hline Tween $^{\circledR} 40$ emulsion & $4.7 \pm 0.0$ & $2.2 \pm 0.0$ & $60.0 \pm 0.0$ & $3.2 \pm 0.0$ & $19.2 \pm 0.0$ & $8.1 \pm 0.0$ & $0.6 \pm 0.0$ & $1.0 \pm 0.0$ \\
\hline Oil body emulsion (unheated seeds) & $4.4 \pm 0.0$ & $1.9 \pm 0.0$ & $56.1 \pm 0.1$ & $3.7 \pm 0.0$ & $20.8 \pm 0.0$ & $11.4 \pm 0.0$ & $0.6 \pm 0.0$ & $1.2 \pm 0.0$ \\
\hline Oil body emulsion (heated seeds) & $4.3 \pm 0.0$ & $1.8 \pm 0.0$ & $56.5 \pm 0.1$ & $3.2 \pm 0.0$ & $20.9 \pm 0.1$ & $11.8 \pm 0.0$ & $0.5 \pm 0.0$ & $1.1 \pm 0.0$ \\
\hline
\end{tabular}




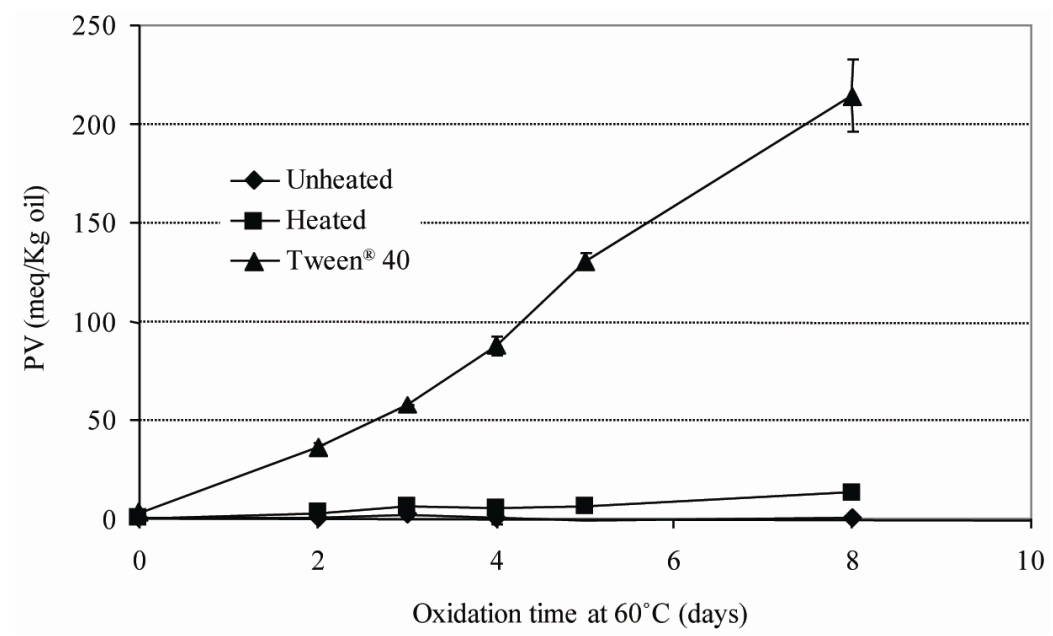

Figure 2. Change in oil peroxide value (PV) during accelerated oxidation $\left(60^{\circ} \mathrm{C}\right.$, dark) of aqueous dispersions $(5 \%$ o/w) of canola oil bodies extracted from heated and unheated seed and an emulsion prepared from refined canola oil and Tween ${ }^{\circledR} 40$ emulsifier.

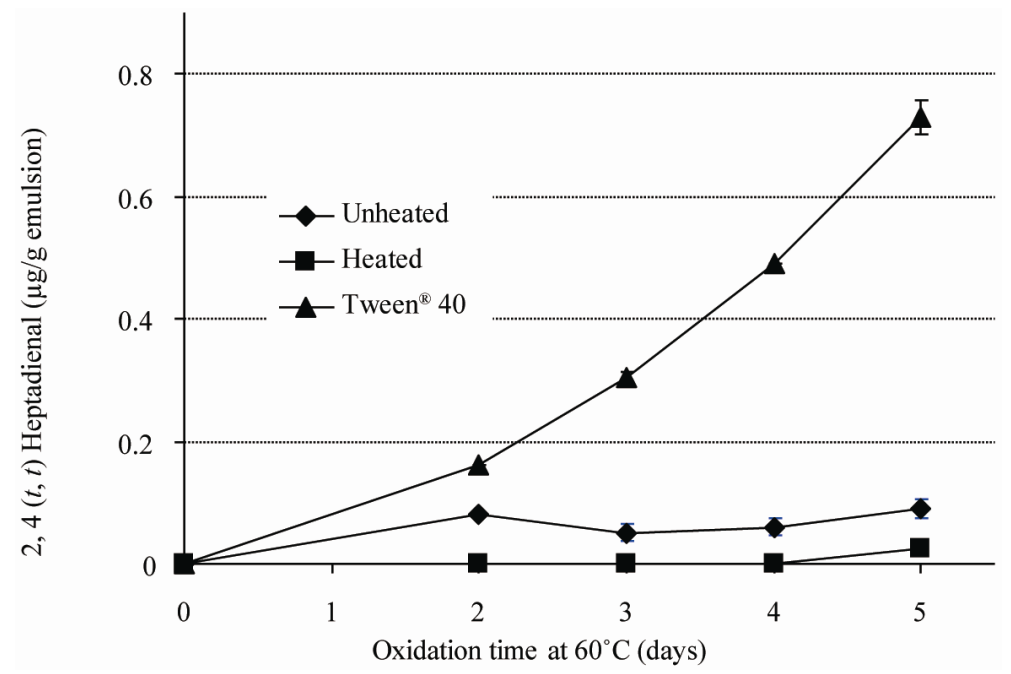

Figure 3. Development of trans, trans 2,4 -heptadienal during accelerated oxidation $\left(60^{\circ} \mathrm{C}\right.$, dark) of aqueous dispersions $(5 \%$ $\mathrm{o} / \mathrm{w}$ ) of canola oil bodies extracted from heated and unheated seed and an emulsion prepared from refined canola oil and Tween $^{\circledR} 40$ emulsifier.

lenic acids during accelerated oxidation (Figure 4). Linolenic acid being the most unsaturated fatty acid of canola oil is the constituent of canola that is most susceptible to oxidative degradation. The content of linolenic acid in the canola oil/Tween ${ }^{\circledR} 40$ emulsion fell rapidly with less than $20 \%$ of the original amount remaining after 8 days of oxidation. In contrast, there was no discernable change in the linolenic acid content in the canola $\mathrm{OB}$ emulsions during the entire observation period (8 days of accelerated oxidation). The depletion of linoleic acid in the canola oil/Tween ${ }^{B} 40$ emulsion followed the same pattern as that of linolenic acid, albeit at a slower rate.

The above results (Figures 2-4) clearly demonstrate the superior oxidative stability of emulsions prepared from intact canola OBs compared with emulsions prepared from refined canola oil and emulsifier. More importantly, there was no discernible difference between the oxidative stabilities of emulsions prepared from OBs extracted from the heated and unheated canola seed, suggesting that no additional protection is provided to the OBs by phenolic antioxidants over and above the natural protection inherent in intact OBs by virtue of its structural integrity. The reason for this is apparent from the distribution of phenols among various fractions during aqueous extraction of OBs from the unheated canola seed. The OBs extracted from the unheated seed retained only $4.5 \%$ $(\mathrm{w} / \mathrm{w})$ of the phenols originally present in the seed, with just $0.26 \%(\mathrm{w} / \mathrm{w})$ transferring to the TAG component of the OBs (Figure 5). A major portion $(59.8 \%, w / w)$ of 


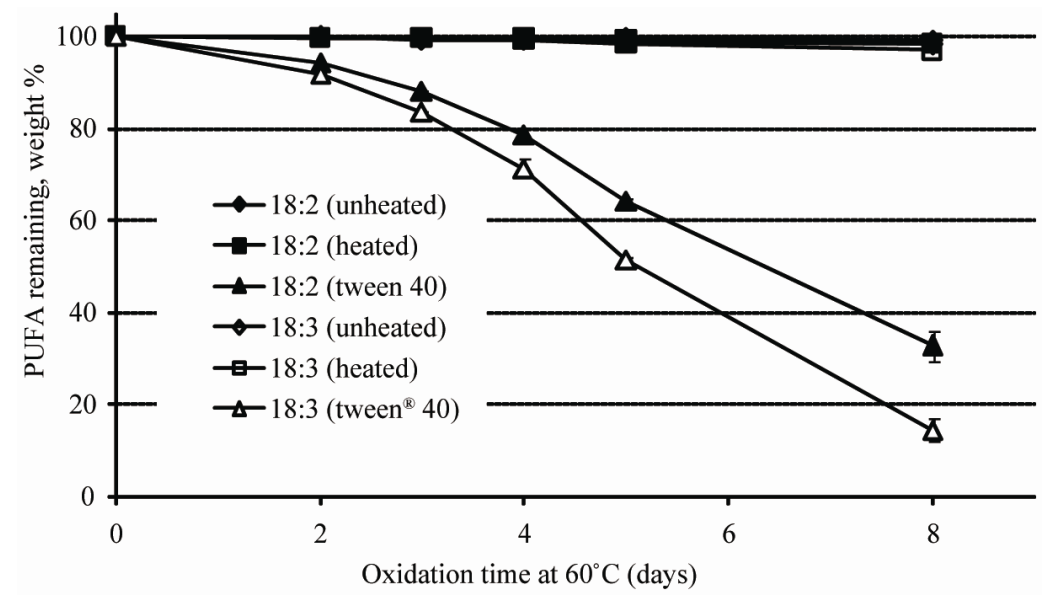

Figure 4. Depletion of linoleic and linolenic acid contents, expressed as the percentage remaining, during accelerated oxidation $\left(60^{\circ} \mathrm{C}\right.$, dark) of aqueous dispersions $(5 \% \mathrm{o} / \mathrm{w})$ of canola oil bodies extracted from heated and unheated seeds and an emulsion prepared from refined canola oil and Tween ${ }^{\circledR} 40$ emulsifier.

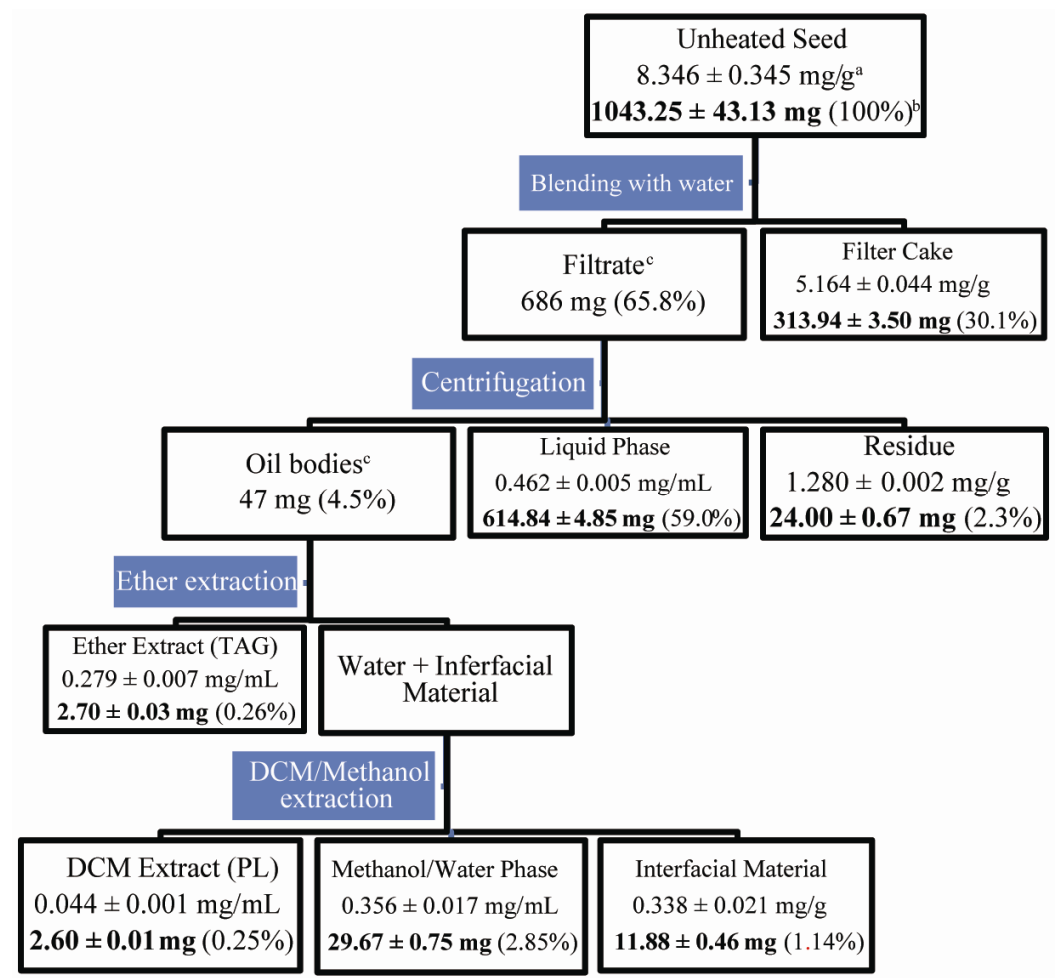

${ }^{\mathrm{a}}$ Concentration of phenols expressed as gallic acid equivalents; ${ }^{\mathrm{b}}$ Amount of phenols (the amount of phenol expressed as a percentage of the total amount in seed is shown in parenthesis); ${ }^{c}$ Indicates that the concentration was measured indirectly from component concentrations.

Figure 5. Transfer of phenolic compounds from unheated canola seed to oil bodies, oil body components and waste during typical aqueous extraction of canola oil bodies. Phenol contents measured directly are highlighted; others were calculated from values measured directly. The results shown are the average values based on $125 \mathrm{~g}$ lots of seed, analysed in triplicate.

the phenols was collected in the liquid phase after centrifugal separation of the OBs with another significant portion $(30.1 \%, \mathrm{w} / \mathrm{w})$ retained in the filter cake. A similar trend in phenol partition was observed for the heattreated seed (Figure 6); the OBs extracted from heattreated seed contained $0.9 \%(\mathrm{w} / \mathrm{w})$ of the original phe- nols while the liquid phase after centrifugal separation of OBs contained $46.9 \%(\mathrm{w} / \mathrm{w})$.

It is noteworthy that $51.0 \%(\mathrm{w} / \mathrm{w})$ of the original phenol content was retained in the filtrate from the seed/ water blend of the heat-treated seed in contrast to $65.8 \%$ $(\mathrm{w} / \mathrm{w})$ that was retained in the corresponding filtrate from 


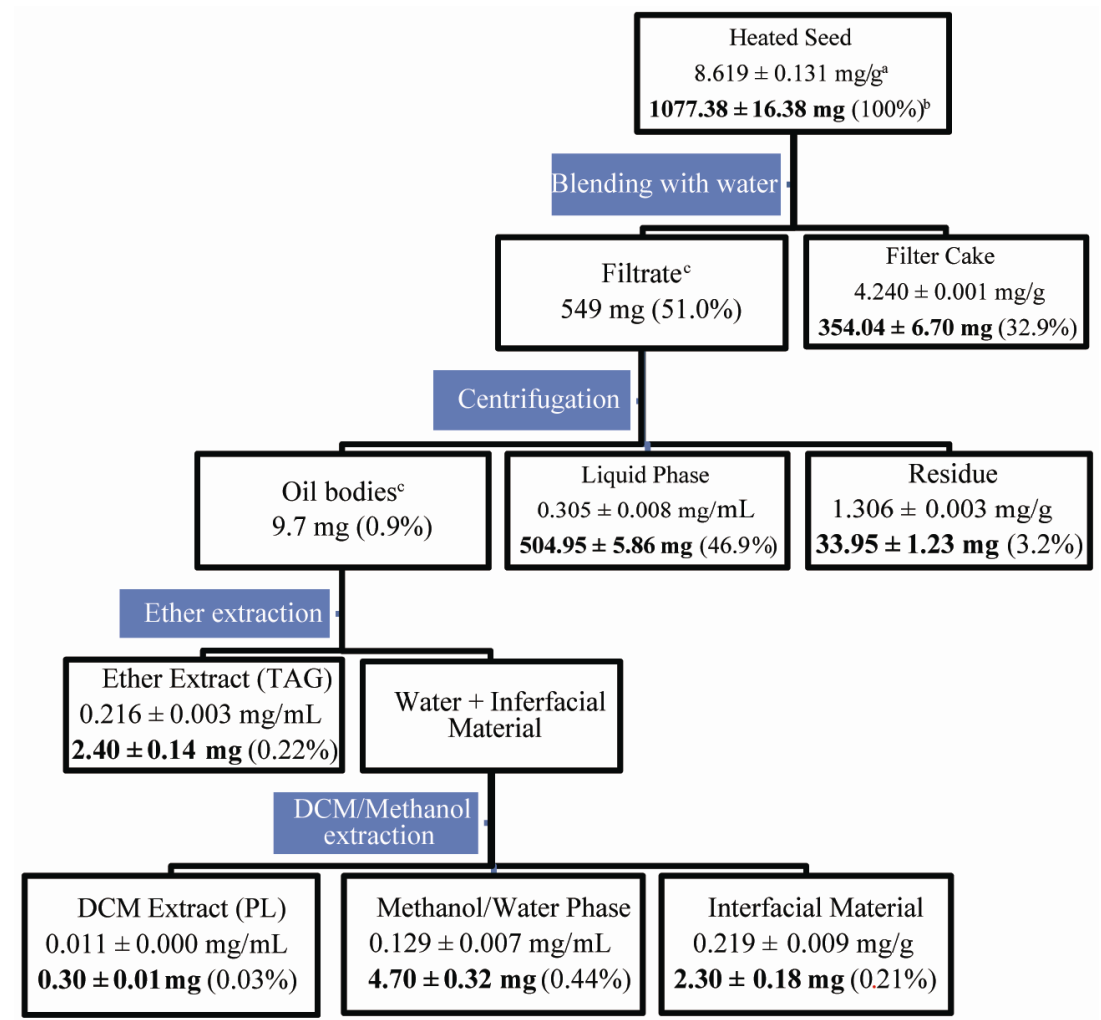

${ }^{a}$ Concentration of phenols expressed as gallic acid equivalents; ${ }^{b}$ Amount of phenols (the amount of phenol expressed as a percentage of the total amount in seed is shown in parenthesis); ${ }^{c}$ Indicates that the concentration was measured indirectly from component concentrations.

Figure 6. Transfer of phenolic compounds from heat-treated canola seed to oil bodies, oil body components and waste during typical aqueous extraction of canola oil bodies. Phenol contents measured directly are highlighted; others were calculated from values measured directly. The results shown are the average values based on $\mathbf{1 2 5} \mathbf{g}$ lots of seed, analysed in triplicate.

the unheated seed. This implies that appreciably more phenols remained in the filter cake from heat-treated seed - a result that could be attributed to the known tendency of phenols to bind more strongly to heat-denatured protein $[27,28]$. We could account for $95.9 \%(\mathrm{w} / \mathrm{w})$ of the phenols originally present in the unheated but only $81.1 \%$ $(\mathrm{w} / \mathrm{w})$ of those present in the heat-treated seed. The lower recovery of phenols from the heat-treated seed could be attributed to incomplete extraction in to $70 \%$ ethanol due to complexation with heat-denatured protein, thus giving lower values by the Folin-Ciocalteu assay.

It is noteworthy that the OBs extracted from canola seed captured only a small portion $(0.9 \%-4.5 \%$, w/w) of the phenols originally present in the seed irrespective of whether the seed was subjected to a prior heat-treatment or not. Phenolic compounds in mature canola (Brassica napus) seed have been reported to occur in the form of various deposits located at the border of the cytoplasm and the cell wall and between the plasmalemma and the cell wall of the othermost columella layer rather than within OBs, and the largest amount of phenolic compounds is found within the protein bodies [29]. Our results showed that the bulk of these phenolic compounds are removed with the storage proteins in the water washings and residues, and are only sparingly transferred to the extracted OBs.

In addition to the phenolic compounds derived from sinapine, canola contains another group of phenolic antioxidants, i.e. tocopherols. In sunflower seed, tocopherols are reported to be intrinsic components of seed OBs [30]. The total tocopherol content in Australian grown canola has been reported to vary widely $(256-928 \mu \mathrm{g} / \mathrm{g}$ oil) depending on the genotype and growing environment [31]. The tocopherol content in canola seed remains more or less unchanged after heat treatment $\left(165^{\circ} \mathrm{C}\right)$ [22], and thus would have equally contributed to the oxidative stability of OBs extracted from heated and unheated canola seed if they were intrinsic components of the OBs as has been reported for sunflower [30]. Although we did not specifically measure the content of tocopherol in the OBs extracted from canola seed (125 g seed, Figures 5 and 6), the small amounts of phenolic compounds found in the OBs from unheated and heated canola seed $(47 \mathrm{mg}$ and $9.7 \mathrm{mg}$, respectively) could have accounted for some or all of the tocopherol (approximately $10 \mathrm{mg}$ ) estimated to be present in the amount of seed used for OB extraction. 
In this event the natural tocopherols could have further enhanced the oxidative stability of OBs extracted from both heated and unheated seed.

\section{Conclusion}

The exceptional stability of emulsions prepared from intact canola OBs against coalescence and lipid oxidation raises the prospect of using natural canola $\mathrm{OB}$ extracts for producing stable food emulsions. Many food emulsions are produced using synthetic emulsifiers and antioxidants. As shown in the present study, stable oil in water emulsions can be prepared from natural OBs without the use of external emulsifiers or antioxidants, thus paving the way for the potential production of totally natural food emulsions. In an environment where there is increasing consumer preference for foods free from synthetic additives, the ability to produce stable emulsions using strictly natural components would be beneficial to the food industry. The sinapine-derived phenolic antioxidants occurring in canola seed are incapable of enhancing the oxidative stability of extracted canola OBs as they are almost completely removed by the aqueous extraction process typically used for $\mathrm{OB}$ extraction.

\section{Acknowledgements}

We thank Lucky Inturrisi of Cargill Australia for canola seed and oil, Thomas Boiteau for SDS-PAGE analysis.

\section{REFERENCES}

[1] A. H. C. Huang, "Oleosins and Oil Bodies in Seeds and Other Organs," Plant Physiology, Vol. 110, No. 4, 1996, pp. 1055-1061. doi:10.1104/pp.110.4.1055

[2] F. Capuano, F. Beaudoin, J. A. Napier and P. R. Shewry, "Properties and Exploitation of Oleosins," Biotechnology Advances, Vol. 25, No. 2, 2007, pp. 203-206. doi:10.1016/j.biotechadv.2006.11.006

[3] J. T. C. Tzen, Y. Z. Cao, C. Ratnayake and A. H. C. Huang, "Lipids, Proteins and Structure of Seed Oil Bodies from Diverse Species," Plant Physiology, Vol. 101, No. 1, 1993, pp. 267-276.

[4] A H. C. Huang, "Structure of Plant Seed Oil Bodies," Current Opinion in Structural Biology, Vol. 4, No. 4, 1994, pp. 493-498. doi:10.1016/S0959-440X(94)90210-0

[5] T. C. Tzen and A. H. C. Huang, "Surface Structure and Properties of Plant Seed Oil Bodies," Cell Biology, Vol. 117, No. 2, 1992, pp. 327-335. doi:10.1083/jcb.117.2.327

[6] P. Jolivet, C. Boulard, A. Bellamy, C. Larre, M. Barre, H. Rogniaux, S. D'andrea, T. Chardot and N. Nesi, "Protein Composition of Oil Bodies from Mature Brassica napus Seeds," Proteomics, Vol. 9, No. 12, 2009, pp. 3268-3284. doi:10.1002/pmic.200800449

[7] A. H. C. Huang, "Oil Bodies and Oleosins in Seeds," Annual Reviews Plant Physiology Plant Molecular Biology, Vol. 43, 1992, pp. 177-200. doi:10.1146/annurev.pp.43.060192.001141

[8] Z. Purkrtova, P. Jolivet, M. Miquel and T. Chardot, "Sturcture and Function of Seed Lipid Body-Associated Proteins," C.R. Biologies, Vol. 331, No. 10, 2008, pp. 746754. doi:10.1016/j.crvi.2008.07.016

[9] D. A. Priestley and A. C. Leopold, "Absence of Lipid Oxidation during Accelerated Aging of Soybean Seeds," Plant Physiology, Vol. 63, No. 4, 1979, pp. 726-729. doi:10.1104/pp.63.4.726

[10] D. A. Priestley and A. C. Leopold, "Lipid Changes during Natural Aging of Soybean Seeds," Plant Physiology, Vol. 59, 1983, pp. 467-470.

doi:10.1111/j.1399-3054.1983.tb04231.x

[11] J. Lee, R. Welti, M. Roth, W. T. Schapaugh, J. Li and H. N. Trick, "Enhancecd Seed Viability and Lipid Compositional Changes during Natural Aging by Suppressing Phospholipase Da in Soybean Seed," Plant Biotechnology Journal, Vol. 10, No. 2, 2012, pp. 164-173. doi:10.1111/j.1467-7652.2011.00650.x

[12] I. D. Fisk, D. A. White, M. Lad and D. A. Gray, "Oxidative Stability of Sunflower Oil Bodies," European Journal of Lipid Science and Technology, Vol. 110, No. 10, 2008, pp. 962-968. doi:10.1002/ejlt.200800051

[13] D. A. Gray, G. Payne, D. J. McClements, E. A. Decker and M. Lad, "Oxidative Stability of Echium Plantagineum Seed Oil Bodies," European Journal of Lipid Science and Technology, Vol. 112, No. 7, 2010, pp. 741-749. doi:10.1002/ej1t.200900280

[14] US Department of Health and Human Services, "Dietary Guidelines for Americans 2010," Washington DC, USDA.

[15] M. Naczk, F. Shahidi and A. Sullivan, "Current Research Developments on Polyphenolics of Rapeseed/Canola: A Review," Food Chemistry, Vol. 62, No. 4, 1998, pp. 489502. doi:10.1016/S0308-8146(97)00198-2

[16] C. Wijesundera, C. Ceccato, P. Fagan and S. Shen, "Seed Roasting Improves the Oxidative Stability of Canola ( $B$. Napus) and Mustard (B. Juncea) Seed Oils," European Journal of Lipid Science and Technology, Vol. 110, No. 4, 2008, pp. 360-367. doi:10.1002/ejlt.200700214

[17] A. Spielmeyer, A. Wagner and G. Jahreis, "Influence of Thermal Treatment of Rapeseed on Canolol Content," Food Chemistry, Vol. 112, No. 4, 2009, pp. 944-948. doi:10.1016/j.foodchem.2008.07.011

[18] A. Koski, S. Pekkarinen, A. Hopia, K. Wahala and M. Heinonen, "Processing of Rapeseed Oil: Effects on Sinapic Acid Derivative Content and Oxidative Stability," European Food Research and Technology, Vol. 217, No. 2, 2003, pp. 110-114. doi:10.1007/s00217-003-0721-4

[19] W. W. Christie, "Preparation of Lipid Extracts from Tissues," In: W. W. Christie, Ed., Advances in Lipid Methodology, Oily Press, Dundee, 1993, pp. 195-213.

[20] E. G. Bligh and W. J. Dyer, "A Rapid Method of Total Lipid Extraction and Purification," Canadian Journal of Physiology and Pharmacology, Vol. 37, 1959, pp. 911917. doi:10.1139/y59-099

[21] S. Vuorela, A. Meyer and M. Heinonen, "Quantitative Analysis of the Main Phenolics in Rapeseed Meal and Oils Processed Differently Using Enzymatic Hydrolysis 
and HPLC," European Food Research and Technology, Vol. 217, No. 6, 2003, pp. 517-523.

doi:10.1007/s00217-003-0811-3

[22] D. Wakamatsu, S. Morimura, T. Sawa, K. Kida, C. Nakai and H. Maeda, "Isolation, Identification, and Structure of a Potent Alky-Peroxyl Radical Scavenger in Crude Canola Oil, Canolol," Bioscience Biotechnology Biochemistry, Vol. 69, No. 8, 2005, pp. 1568-1574. doi:10.1271/bbb.69.1568

[23] A. Spielmeyer, A. Wagner and G. Jahreis, "Influence of Thermal Treatment of Rapeseed on the Canolol Content," Food Chemistry, Vol. 112, No. 4, 2009, pp. 944-948. doi:10.1016/j.foodchem.2008.07.011

[24] M. Li, L. J. Smith, D. C. Clark, R. Wilson and D. J. Murphy, "Secondary Structure of a New Class of Lipid Body Protein from Oilseeds," Journal of Biological Chemistry, Vol. 267, No. 12, 1992, pp. 8245-8253.

[25] A. Richards, M. Golding, C. Wijesundera and L. Lundin, "The Influence of Secondary Emulsifiers on Lipid Oxidation within Sodium Caseinate-Stabilized Oil-in-Water Emulsions," Journal of American Oil Chemists' Society, Vol. 88, No. 1, 2011, pp. 65-73.

[26] E. N. Frankel, "Lipid Oxidation," 2nd Edition, The Oily Press, Bridgewater, 2005.
[27] D. A. Rickert, L. A. Johnson and P. A. Murphy, "Improved Fractionation of Glycinin and B-Conglycinin and Partitioning of Phytochemicals," Journal of Food and Agriculture, Vol. 52, No. 6, 2004, pp. 1726-1734. doi:10.1021/jf035248x

[28] F. Speroni, V. Milesi and M. C. Añón, "Interactions between Isoflavones and Soybean Proteins: Applications in Soybean-Protein Isolate Production," LWT Food Science and Technology, Vol. 43, No. 8, 2010, pp. 1265-1270. doi:10.1016/j.lwt.2010.03.011

[29] M. Stefanowska, A. M. Zobel and M. Kuras, "Cytochemical Localization of Phenolic Compounds in Columella Cells of the Root Cap during Maturation of Seeds on Brassica napus L.," Plant Biology, Vol. 5, No. 4, 2003, pp. 378-382. doi:10.1055/s-2003-42714

[30] I. D. Fisk, D. A. White, A. Carvalho and D. A. Gray, "Tocopherol-An Intrinsic Component of Sunflower Seed Oil Bodies," Journal of American Oil Chemists' Society, Vol. 83, No. 2, 2006, pp. 341-344.

[31] A. Richards, C. Wijesundera and P. Salisbury, "Genotype and Growing Environment Effects on the Tocopherol and Fatty Acids of Brassica napus and B. juncea," Journal of American Oil Chemists' Society, Vol. 85, No. 2, 2006, pp. 159-168. 